\title{
ICTs Supportive and Therapeutic Contribution in Psychoemotional Disorders in Childhood and Adolescence
}

\author{
https://doi.org/10.3991/ijes.v6i2.8796 \\ Bakola N. Lizeta $(\bowtie)$, Rizos D. Nikolaos, Athanasios S. Drigas \\ National Center for Scientific Research "Demokritos", Agia Paraskevi, Greece \\ lizeta79@gmail.com
}

\begin{abstract}
This paper aims at reviewing the supportive and therapeutic contribution of ICTs on the most common psychoemotional disorders of childhood and adolescence. The various approaches to classifying behavioral and emotional dysregulations in children and adolescents have been reviewed and the boundaries between normative variation and clinically significant presentations have been determined. Furthermore, we present the major categories of mental illnesses in children and adolescents investigating the etiology, the diagnostic criteria, the epidemiology and treatments by critically reviewing the outcomes of relevant studies. The findings indicate that significant steps have been made in this field, but it is vital to underline the need for more extended investigation in school aged children and adolescents for this purpose.
\end{abstract}

Keywords-Psychoemotional Disorders, Childhood, Adolescence, ICT

\section{$1 \quad$ Introduction}

Many psychological disorders firstly diagnosed in children involve the combination of physiological and genetic components. The term psychological disorders is sometimes used to refer to the most frequently known as mental disorders or psychiatric disorders (K. Cherry). Mental illnesses or disorders are patterns of behavioral or psychological symptoms that impact multiple areas of life.

In this review we present some of the major categories of disorders described in the Diagnostic and Statistical Manual of Mental Disorders (DSM), which is the most widely, used system for classifying mental disorders and provides standardized diagnostic criteria. Furthermore, we investigate the contribution and the importance of ICTs in children and adolescents with mental illnesses. The role that Information and Communication Technologies (ICTs) play in many educational and behavioral aspects is undisputable. There are many treatments and interventions based on the Internet and aiming at mental disorders and psychological problems. 


\section{Major Categories of Mental Illnesses}

\subsection{Anxiety Disorders}

Generalized Anxiety Disorder (GAD): Susan J. Bradley (2001) in this paper presents anxiety and mood disorders as the most common disorders in children and adolescents, who presage later emotional difficulties and disabilities. According to statistics, she indicated that depression also affects children and adolescents although it is less common in younger children. The researcher supports that boys and girls are equally affected despite the fact that adolescent girls are much more vulnerable. Moreover, suicidal ideation is found to affect about $50 \%$ of youth, but the actual suicide attempts are less common and more frequently in female adolescents even though males are more likely to commit suicide. In addition, the study showed that dysthymic condition seems to be connected with affective disorders in children. The etiology is multifactorial and includes genetic factors, the stress reactivity and individual specific environmental factors such as family and the child's ordinal position, but also sex and child's temperament (DSM-IV/DSM-PC). Treatment goals include stress reduction, exposure to the avoided or feared situation and specific strategies like: CBT, IPT, SSRLs, Behavioral Family Therapy [1].

Mogg, et al. [2] conducted a study to enrich previous research demonstrating that anxiety is associated with poor control of executive attention. For this purpose, children from a community sample were drawn and separated to three different groups, as in children with anxiety disorders (other than specific phobias), ADHD and no disorder. They were exhibited to some tests supported from the ICTC's by using DAWBA and CBCL for the psychiatric and psychopathology diagnoses respectively, as much as Weschler Intelligence Scale for children for the IQ estimation and Attention Network Task (ANT) to assess executive attention, alerting and orienting. The paper concludes that executive attention is impaired in children with anxiety disorder (excluding specific phobias), as well as in children with ADHD, relative to disorder- free children. Furthermore, executive attention was less efficient in disorder-free children who have high, relative to low levels of anxiety symptoms [2].

Furthermore, Stefan Scherer et al. exploited the contribution of ICTS', introduced a dataset, named Distress Assessment Interview Corpus (DAIC) to verify the strong correlation between nonverbal behaviors and specific psychological disorders, particularly distress, depression, anxiety and post-traumatic stress disorder. For this purpose, the DAIC uses automatic behavior descriptors and manual annotations. The behavior descriptors are vertical head and eye gaze, smile intensity and duration, whereas the manual annotations refer to self-adaptors and legs fidgeting. The main findings confirm the downward trend of the face and eye gaze of people with the above disorders, the reduced intensity and shorter duration of smiles and the longer exhibition on self-touches and fidgets [3].

Panic Disorder (with and without Agoraphobia): Agoraphobia is common in children and adolescents. Crozier et al. report that according to the DSM-IV-TR, agoraphobia can occur with or without the presence of panic disorder and is defined by extreme anxiety as panic attack or panic-like symptoms, in specific situations 
where the person feels trapped or far from help. This anxiety also usually leads to phobic avoidance. The researchers present the conclusions of two different studies of US and German adolescents where they found that agoraphobia constitute the most frequently occurring anxiety disorder and that the adolescents who had never experienced a panic attack were only moderately more likely to develop subsequent agoraphobia.

Phobias (including Specific Phobia, Disgust Sensitivity, Social Phobia, also called Social Anxiety Disorder):

Specific Phobia: Marjorie Crozier et al. exhibit the description, the epidemiology, the genetic patterns and the subtypes of specific phobias. As it is referred in the study, specific phobias are the most prevalent anxiety disorder (Kessler et al., 2005) without comorbid diagnoses (Costello \& Angold, 1995) and contain intense fears of specific objects or situations including animals, heights, enclosed spaces, or darkness. To distinguish phobias from typical fears in childhood the phobia diagnosis should be considered when the fear is excessive and causes marked interference in child's life and is present for at least 6 months (Crozier et al. 2011).Additionally, according to DSM-IV-TR, they should be diagnosed when the symptoms are not better explained by other mental disorders such as OCD, PTSD, SP, PD. The researchers observe cultural, age and gender differences between people with specific phobias. They also examined the factor structure of all specific phobias and found the following factors: Agorophobia, Speaking, High/Water, Being observe, Threat and supplementary, Social Fears and Specific Fears. Finally, this study did find common genetic factors contributing to all phobias.

Disgust Sensitivity: Disgust Sensitivity is defined as a predisposition to experiencing disgust in a wide variety of settings and this sensitivity has been proposed to contribute to the development of a variety of disorders such as blood-injection-injury phobias, animal phobias and OCD (Olatunji \& Deacon, 2008). According to the researchers, feelings of disgust in individuals with phobias are detected more than fear. As a conclusion, Crozier et al. mention that disgust sensitivity is associated with genetic and environmental factors and that there is also a correlation between gender and specific phobias.

Social Phobia: Social Phobia is described as an intense fear or discomfort in social situations, which can be limited to one specific factor or be generalized to all social settings (Crozier et al., 2011). The fear of embarrassment and the overestimated perception of how anxious the individuals appear physically are referred as some of the causes of this type of anxiety. All the criteria and symptoms are included in DSM-IVTR (pp. 416-417) and they are slightly varied between children and adolescents. It is mentioned that children and adolescents with social phobia are at a high risk for developing substance use and major depression and educational problems. Pertaining to the epidemiology it is detailed that different studies have chosen to categorize common types of fears differently and inconsistent findings have been reported for prevalence rates and gender differences in social phobia [4].

Gutierrez-Maldonado et al, from the University of Barcelona, decided to perform a study to test the efficacy of a treatment by using a new type of exposure technique for school phobia, as an alternative or a complement to the traditional forms of vivo ex- 
posure, and that is the exposure by means of virtual reality techniques. The research group has developed a series of virtual reality environments, which can be integrated in a treatment program for children and adolescents with school phobia. This study considered 36 children with a mean age of 11 years old, who were divided into 2 groups and 18 of them being with high scores on school phobia. Instruments used for the assessment of participants were: IME, FSSC-R, SRAS-C, STAI -C. A system of virtual environments was developed (School, Classrooms) and applied on PC systems with objects, animations, avatars. The results show that this treatment reduces the intensity of school phobia and proved that the use of ICTS' and more specific it's of virtual reality exposure is an important tool among the rest of techniques for the treatment of school phobia [5].

Post-Traumatic Stress Disorder (PTSD): Bruce D. Perry reviews childhood PTSD, especially from a neurobiological aspect. PTSD is characterized by recurring intrusive flashbacks, persistent avoidance of traumatic stimuli, hyperactivity, and irritability. It is also experienced by abuse victims, neglected persons, survivors of disasters and witnesses to violence. Studies conducted at the Center for the Study of Childhood Trauma in Chicago indicate that family history of psychiatric disorder and the age of trauma occurrence are two key factors. Furthermore, a stressful situation results in neurophysiological changes in locus coeruleus, ventral tegmental nucleus activity and in catecholamine systems with a significant impact on brain development. Research also proved that tachycardia is a feature of PTSD, while alpha 2-adrenergic receptors and clonidine could be effective stress medications [6].

Separation Anxiety Disorder: Peter Lewinsohn et al. conducted a study to identify the relationship between childhood separation anxiety disorder (SAD) and psychopathology during young adulthood (ages 19-30). SAD is a disorder of childhood characterized by the subject's reluctance to be separated from major attachment figures because he/she fears that something awful will happen to this figure. The disorder has an onset age of approximately 7 years and a duration of about 3 years. The researchers used 816 participants of the Oregon Adolescent Depression Project (OADP) as a sample and the subjects were divided into 4 groups: SAD, other anxiety disorders, a heterogeneous psychiatric disorders control group and a not mentally ill control group. The results based on ICTS' support confirmed that childhood SAD is a strong risk factor $(78,6 \%)$ for the development of internalizing disorders in young adulthood, especially for panic disorder and depression, and confers a decreased risk for externalizing disorders, such as alcoholism and substance use [7].

Nightmares: In his first systematic study, conducted by Mehmet Yucel Agargun et al., to investigate the relationship between nightmares and dissociative experiences by considering the role of childhood traumatic events in adolescents as well as whether the severity of dream anxiety is correlated with dissociative experiences in subjects with and without traumatic events. 292 undergraduate students from the Yuzuncu Yil University at Van City participated in this study. All subjects were informed from the beginning about the difference between nightmares and night terrors. The instruments they used contained: a self-administered questionnaire, a structured interview for childhood traumatic events, including childhood physical or sexual abuse, maternal loss, and maternal separation, the Van Dream Anxiety Scale (VDAS) to establish a 
reliable and valid measure of dream anxiety, the Dissociative Experiences Scale (DES) for the overall psychometric properties and finally the Spearman correlation analysis, taken advantage of the ICTS' use, to establish the correlation between the VDAS and the DES scores. According to the results, females were more vulnerable to nightmares than males and the subjects who had experienced at least one childhood traumatic event also had higher mean DES score than those who had not. As a conclusion, the article certifies that there is a relationship between nightmares and dissociative experiences and that childhood traumatic events may play a key role in this association [8].

\section{Obsessive-Compulsive Disorder (OCD)}

In their article, I.Heyman, E.Fombonne et al. presented a British study of OCD in children and adolescents in order to establish the prevalence and associates of the disorder in these ages. For the purposes of the study, 10.348 children were assessed and the Development and Well-Being Assessment (DAWBA) was used with summary sheets based on DSM-IV and ICD-10 as well. The children divided into three subjects with normal controls, emotional disorders and OCD and of the whole sample, 25 received a diagnosis of OCD $(0.25 \%)$. The findings of the survey reveal that OCD occurred equally in boys and girls, its rates rise with increasing age, it is associated with lower socio-economic class and intelligence and, finally, that there are high rates of comorbidity [9].

\section{$4 \quad$ Mood Disorders (Affective Disorders)}

Hamish Fulford et al. conducted a systematic review and metasynthesis of all qualitative studies regarding the use of ICTs by people with mood disorders. ICTs, specifically health informatics applications (e-Health), provide patients with mood disorders with new ways to manage their health in an accessible, interactive and instant way. Such interventions are computerized cognitive behavioral therapy (CCBT), information websites, online counseling, forums, self-management groups and social networking sites (SNSs). People are motivated to use ICTs because of their affordability, accessibility (especially for those living in remote or rural areas), versatility and functionality. Of utmost importance is, moreover, the privacy and anonymity, the decrease of feelings of stigma and, in terms of social relationships, the fact that they facilitate dialogue, collaboration and opportunities for feelings and experiences exchange [10].

Herve Javelot, Anne Spadazzi et al., in their paper, presented the PSYCHE project (Personalised monitoring Systems for Care in mental Health). This in an interactive monitoring system based on portable devices, which collect multivariable multiparametric data from patients with mood disorders. It consists of two management systems- the knowledge management system and the disease management system- and involves five contributors. The researchers describe the function of this ICTS' system, presenting that the data are sent to the knowledge management system through a 
WWS and a smartphone, while a garment, composed of textile interfaces, collects physiological signals about mood states during day and night [11].

\section{$5 \quad$ Psychotic Disorders}

\section{$5.1 \quad$ Schizophrenia}

Alan S. Brown (2008), in his editorial, investigated the possibility that infections during childhood and adulthood could be risk factors for schizophrenia. The use of maternal biomarkers in birth cohorts helped to validate infections in pregnancies and it is highlighted that infections during prenatal and postnatal period can result in neuropsychiatric disorders. Moreover, a study in Sweden revealed a 50\% higher risk of non-affective psychosis among individuals exposed in childhood to viral CNS infection, but no increased risk for those exposed to bacterial CNS infections. Other studies have also shown elevated serum levels of antibody to $\mathrm{T}$. gondii in patients with schizophrenia and neurobehavioral disturbances during such infection [12].

\section{Dementias}

Susan Greenfield is referred to "Digital Dementia", a term invented by neuroscientist Manfred Spitzer to describe the results from the catachresis of digital technology which cause malfunction of cognitive abilities. Previous research has found a potential connection between action video gaming and the risk for psychological disorders, like dementia. Through the navigation in a virtual maze associated with the hippocampus, it was proved that the excessive use of video games may cause mental breakdown. The conclusion is that the reduction of grey matter in the hippocampus is related to an increased risk for Psychoemotional Disorders [13].

\section{$7 \quad$ Eating Disorders}

Kenisha Campell and Rebecka Peebles present a brief description of Eating Disorders in children and adolescents. These disorders include anorexia nervosa (AN), bulimia nervosa $(\mathrm{BN})$ and other EDs not otherwise specified (EDNOS). The prevalence and mortality rates differ and are now seen in younger children, boys and minority groups. Genetic and biological predispositions, but also environmental, sociocultural and psychological reasons seem to be the main etiology. Studies show that patients with EDs present with gaining or losing weight problems, growth delay, abnormal eating behaviors, over-exercise and body image concerns. Organic, neurological and psychiatric problems also arise. Treatment modalities (individual therapies, cognitive behavioral therapies, family-based therapies) and pharmacotherapy aim at multifaceted recovery and, finally, primary and secondary prevention is critical, especially during childhood and adolescence [14]. 


\section{Impulse Control and Addiction Disorders}

In their paper, Bernardo Dell' Osso et al. present an overview of the impulsive compulsive disorders (ICDs). These are a group of disorders characterized by repetitive pleasurable and gratifying behaviors which cause serious dysfunction in all spheres and failure to resist and inhibit these behaviors. The traditional ICDs are pathological gambling, trichotillomania, kleptomania, pyromania and intermittent explosive disorder, while the new proposed ones include: compulsive-impulsive (CI) Internet usage disorder, C-I sexual behaviors, C-I skin picking and C-I shopping. It is emphasized that there is a close relationship between ICDs and other psychiatric conditions, especially OCD, that is why ICD could be conceptualized as a part of OCD spectrum. The prevalence estimates range and the causes of ICDs can be parental and environmental or even neurochemical predispositions. As far as the treatment is concerned, it includes both pharmacotherapy (SSRIs, mood stabilizers, opioid antagonists) and psychotherapy (CBT, self-help groups, family therapy, social skill training) [15].

Susana Jimenez -Murcia et al, are referred to Playmancer (ICT European initiative), a new serious videogame that was created as an additional therapy tool for treating Mental Disorders. The purpose of this presentation is to analyze the efficiency and effectiveness of this use. 120 patients with Bulimia nervosa and PG participated in a cohort study (patients and controls) and the procedure included two conditions: a standard cognitive - behavioral therapy (CBT) and a weekly exposure in a videogame environment ("Islands") vs. CBT without video game. This was a pilot trial and Playmancer seems to be an innovative approach, which can demonstrate an association between video gaming and physiological and emotional reactions of users. Finally, it is necessary to mention that ICTs and, specifically serious games, have experimented a steady growth of complementing treatments in the health area [16].

Generally, as far as the contribution of ICTs is concerned, J.V. Allen et al. examined the use of telemedicine (interactive televideo) in Pediatric Psychology, a field focused on the psychological and behavioral issues of children and adolescents with chronic illness. Nine studies are detailed and suggest that telemedicine services offer cost-effectiveness, feasibility, reduction of anxiety levels and satisfaction to participants. Such findings are also confirmed by individual, multidisciplinary and group telehealth interventions conducted in the University of Kansas Medical Center [17].

Ed Smeets, in his paper, presents the findings of 2 studies conducted in the Netherlands, which address the role of the school environment in the prevention and management of social, emotional and behavioral difficulties (SEBD) at schools. The key factors are adequate instructional environment, supportive social-emotional climate and a systematic process of identification and evaluation of prevention and intervention strategies. This approach is based on a set of 5 guidelines, which is outlined with respect to the differentiation of learning procedures, use of ICTs and improvement of development and learning progress. In such a system, ICTs facilitate the adaptation of instruction, the monitoring of pupils' progress and information exchange [18]. 


\section{Conclusion}

This review once more makes it clear that psychological, emotional, behavioral problems become more and more common during childhood and adolescence, and that several treatments are used for the handling of these situations and difficulties. Such treatments include, amongst others, Information and Communication Technologies, which combine many advantages and, specifically, accessibility, interaction, privacy and convenient implementation for their patients and their caretakers, thereby making ICTs a substantially efficacious tool.

\section{References}

[1] Bradley, S. J. (2001). Anxiety and mood disorders in children and adolescents: a practice update. Paediatrics \& child health, 6(7), 459-463. https://doi.org/10.1093/pch/6.7.459

[2] Mogg, K., Salum, G. A., Bradley, B. P., Gadelha, A., Pan, P., Alvarenga, P, \& Manfro, G. G. (2015). Attention network functioning in children with anxiety disorders, attentiondeficit/hyperactivity disorder and non-clinical anxiety. Psychological medicine, 45(12), 2633-2646. https://doi.org/10.1017/S0033291715000586

[3] Scherer, S., Stratou, G., Lucas, G., Mahmoud, M., Boberg, J., Gratch, J., \& Morency, L. P. (2014). Automatic audiovisual behavior descriptors for psychological disorder analysis. Image and Vision Computing, 32(10), 648-658. https://doi.org/10.1016/j.imavis. 2014.06.001

[4] Crozier, M., Gillihan, S. J., \& Powers, M. B. (2011). Issues in differential diagnosis: phobias and phobic conditions. In Handbook of child and adolescent anxiety disorders (pp. 722). Springer, New York, NY. McKay Dean, Storch Eric. Handbook of Child and Adolescent Anxiety Disorders 2011 https://doi.org/10.1007/978-1-4419-7784-7 2

[5] Gutiérrez-Maldonado Jose, Magallón-Neri Ernesto, Rus-Calafell Mar, Peñaloza Claudia.Virtual reality exposure therapy for school phobia Anuario de Psicologia 40(2):223 - January 2009

[6] Perry, B. D., \& Azad, I. (1999). Posttraumatic stress disorders in children and adolescents. Current opinion in pediatrics, 11(4), 310-316. https://doi.org/10.1097/00008480199908000-00008

[7] Lewinsohn, P. M., Holm-Denoma, J. M., Small, J. W., Seeley, J. R., \& Joiner, T. E. (2008). Separation anxiety disorder in childhood as a risk factor for future mental illness. Journal of the American Academy of Child \& Adolescent Psychiatry, 47(5), 548-555. https://doi.org/10.1097/CHI.0b013e31816765e7

[8] Agargun, M. Y., Kara, H., Özer, Ö. A., Selvi, Y., Kiran, Ü., \& Kiran, S. (2003). Nightmares and dissociative experiences: The key role of childhood traumatic events. Psychiatry and Clinical Neurosciences, 57(2), 139-145. https://doi.org/10.1046/j.1440-1819.2003.01093.x

[9] Heyman, I., Fombonne, E., Simmons, H., Ford, T., Meltzer, H., \& Goodman, R. (2003). Prevalence of obsessive-compulsive disorder in the British nationwide survey of child mental health. International Review of Psychiatry, 15(1-2), 178-184. https://doi.org/10.1080/0954026021000046146

[10] Fulford, H., McSwiggan, L., Kroll, T., \& MacGillivray, S. (2016). Exploring the Use of Information and Communication Technology by People With Mood Disorder: A System- 
atic Review and Metasynthesis. JMIR mental health, 3(3). https://doi.org/10.2196/mental. 5966

[11] Javelot, H., Spadazzi, A., Weiner, L., Garcia, S., Gentili, C., Kosel, M., \& Bertschy, G. (2014). Telemonitoring with respect to mood disorders and information and communication technologies: overview and presentation of the PSYCHE project. BioMed research international, 2014. https://doi.org/10.1155/2014/104658

[12] Brown, A. S. (2008). The risk for schizophrenia from childhood and adult infections.

[13] Greenfield, S. (2015). Mind change: How digital technologies are leaving their mark on our brains. Random House Incorporated.

[14] Campbell, K., \& Peebles, R. (2014). Eating disorders in children and adolescents: state of the art review. Pediatrics, 134(3), 582-592. https://doi.org/10.1542/peds.2014-0194

[15] Dell'Osso, B., Altamura, A. C., Allen, A., Marazziti, D., \& Hollander, E. (2006). Epidemiologic and clinical updates on impulse control disorders: a critical review. European archives of psychiatry and clinical neuroscience, 256(8), 464-475. https://doi.org/10.1007/ s00406-006-0668-0

[16] Fernández-Aranda, F., Jiménez-Murcia, S., Santamaría, J. J., Gunnard, K., Soto, A., Kalapanidas, E, \& Konstantas, D. (2012). Video games as a complementary therapy tool in mental disorders: PlayMancer, a European multicentre study. Journal of Mental Health, 21(4),

364-374.https://doi.org/10.3109/096382 37.2012 .664302

[17] Van Allen, J., Davis, A. M., \& Lassen, S. (2011). The use of telemedicine in pediatric psychology: Research review and current applications. Child and Adolescent Psychiatric Clinics, 20(1), 55-66. https://doi.org/10.1016/j.chc.2010.09.003

[18] Smeets, E. (2009). Managing social, emotional, and behavioural difficulties in schools in the Netherlands. International Journal of Emotional Education, 1(1), 50-63.

\section{Authors}

L. Bakola is a secondary-school teacher of Greek language. She is also a post graduate student in the programme "Expertise in ICT and Special Education - Psychopedagogy of Integration" and a research associate at N.C.S.R. 'Demokritos', Institute of Informatics and Telecommunications, Net Media Lab.

Agia Paraskevi, 153 10, Athens, Greece.is a primary-school teacher. He is also a post graduate student in the programme "Expertise in ICT and Special Education Psychopedagogy of Integration" and a research associate at N.C.S.R. 'Demokritos', Institute of Informatics and Telecommunications, Net Media Lab, Agia Paraskevi, 153 10, N. Rizos Athens, Greece

A. Drigas is a Research Director and is the founder \& scientific coordinator of Net Media Lab of NCSR "Demokritos" and has been the scientific coordinator of more than 30 National \& international projects on networks and e-services (e-learning, einclusion, e-psychology, e-government, etc). He earned his M.Sc. degree in Elec. Engineering in ICTs from the University of Patras, and his $\mathrm{PhD}$ in Cognitive Science (Memory \& Resource Management in Computer Networks) from the National Technical University of Athens, Greece. In 2000, he earned his bachelor degree in Psychology from the University of Athens, Greece. He is the author of more than 200 publications in international scientific journals and conferences and 8 books. Finally, 
he has been a counselor of many public organizations on ICTs and e-services and nowadays co-organized, launched and teaches at the postgraduate studies program entitled "Expertise in ICT and Special Education-Psychopedagogy of Integration.

Article submitted 27 April 2018. Final acceptance 21 May 2018. Final version published as submitted by the authors. 